\title{
SECRETORY FUNCTION OF THE PROSTATE, SEMINAL VESICLE AND OTHER MALE ACCESSORY ORGANS OF REPRODUCTION
}

\author{
T. MANN \\ A.R.C. Unit of Reproductive Physiology and Biochemistry, \\ University of Cambridge, Cambridge
}

The secretory activity of male accessory organs of reproduction such as the prostate, seminal vesicle, Cowper's gland and similar organs, differs in many ways from that of other secretory organs in the animal body.

\section{DEPENDENGE ON ANDROGENS}

One of the characteristics, which is perhaps most remarkable and in some ways unique, is the specific dependence of the function of organs such as the prostate and seminal vesicle upon the presence of androgenic stimuli. In its extreme form this dependence is clearly reflected in the well documented and much studied set of phenomena of post-castrate involution and testosterone-induced resumption of growth in the accessory organs. Testosterone acts on the male accessory organs directly, without having first to undergo metabolic alteration in the animal body. Convincing proof of such direct action was provided by an experiment recently carried out on bull seminal vesicles in vivo, in which testosterone was injected locally into one seminal vesicle in situ, while the other was left as an untreated control : 3 weeks after local introduction of $10 \mathrm{mg}$ testosterone oenanthate, the treated seminal vesicle was twice as large and contained four times as much fructose and citric acid as the untreated control (Mann, Rowson, Baronos \& Karagiannidis, 1971).

In the course of the same investigation we have also compared the stimulating potency of testosterone with that of $5 \alpha$-dihydrotestosterone, an androgenic steroid to which testosterone is known to be converted in the male accessory organs (Bruchovsky \& Wilson, 1968; Anderson \& Liao, 1968; Wilson \& Gloyna, 1970; Williams-Ashman \& Reddi, 1971). We found that, when introduced directly into the seminal vesicle, dihydrotestosterone oenanthate was capable of promoting growth and formation of fructose and citric acid, but the magnitude of the effect was no more than that of a corresponding dose of testosterone oenanthate. As a matter of fact, in another experiment, in which two castrated twin bulls were injected subcutaneously, one with dihydrotestosterone and the other with testosterone, the latter responded with significantly greater secretory output of fructose and citric acid in electro-ejaculated seminal plasma. However, the growth of the seminal vesicles in both twins was about the same. 
RELATION OF GROWTH TO SEGRETORY FUNCTION IN THE

PROSTATE AND SEMINAL VESIGLE

There are other instances where growth and secretory activity of an accessory organ do not proceed in a parallel fashion. In the case of the human prostate gland, for example, an organ which accounts for most of the high citric acid content of seminal plasma in men, our experience has been that, although a prostate affected by benign hypertrophy or adenocarcinoma continues to secrete citric acid, it does so at a reduced level (Marberger, Marberger, Mann \& Lutwak-Mann, 1962). Our analyses have also shown that not only the cancerous prostate itself, but the metastases of the prostatic adenocarcinoma also continue to secrete citric acid though in concentrations lower than the primary tumor. With regard to the question, as yet poorly understood, why pathological conditions in the prostate affect its growth and secretory activity to a different extent, it may be relevant to point out the observation by Siiteri \& Wilson (1970) that the ratio of $5 \alpha$-dihydrotestosterone/testosterone concentration in human prostates affected by benign hypertrophy can be nearly five times higher than in normal prostate.

Infection is another factor which can also upset the relationship between growth and secretory activity in a male accessory organ of reproduction. In a pubescent bull calf, which had already produced some spermatozoa and had a high concentration of fructose and citric acid in its semen, we observed a rapid decline in both the sperm output and the secretion of fructose and citric acid because of orchitis; when after treatment with antibiotics the inflammation subsided, the fructose and citric acid levels in ejaculates soon returned to normal (Mann, Rowson, Short \& Skinner, 1967). Similarly in men, the acid phosphatase secretion by the prostate, and the fructose secretion by the seminal vesicles, have been shown to decline as a result of inflammatory conditions in the reproductive tract (Eliasson, Molin \& Rajka, 1970).

A situation opposite to the one mentioned earlier, in which the human prostate grows abnormally large without increasing its rate of secretory activity, is occasionally encountered both in man and in animals, either as a result of sexual excitation or following treatment with hormones and certain pharmacological agents. The dog prostate, for example, as Huggins has shown (1945, 1947), normally discharges during intervals between ejaculations not more than $2 \mathrm{ml}$ prostatic secretion/hr; similar amounts, representing the so-called 'resting' or spontaneously voided prostatic secretion, can be obtained from a resting dog by means of a suprapubic prostatic cannula. But following sexual excitation, testosterone injection or administration of pilocarpine, the flow of the prostatic secretion increases rapidly. In a dog injected with pilocarpine, the prostate can discharge as much as $60 \mathrm{ml}$ fluid/hr, that is, something like four times the weight of the organ itself.

\section{CHEMICAL PEGULIARITIES OF MALE AGGESSORY FLUIDS}

In view of the wide fluctuations in the volume of prostatic secretion, depending on whether the fluid was voided spontaneously by a 'resting' prostate or secreted 
by an 'activated' or 'stimulated' organ, it is perhaps not surprising that the chemical composition of the prostatic secretion also shows wide variations, in both inorganic and organic constituents.

For instance, in the 'activated' dog prostatic secretion, potassium ions contribute only about 5 m-equiv. /litre towards total osmolarity, but in the 'resting' secretion about $30 \mathrm{~m}$-equiv./litre (Huggins, 1947). Similar variations affect some of the organic constituents, such as, for example, citrate ions, which represent a substantial contribution to the sum of osmotically active substances in the prostatic secretion.

It is another unusual but very typical feature of the prostatic as well as vesicular secretion in most animal species, that a high proportion of their osmotically active substances is made up by organic compounds. A most outstanding example in this respect, that I came across in my own studies, is the high concentration of inositol in the boar vesicular secretion (Mann, 1954). I was surprised to find that, whilst this secretion contains up to $3 \%$ inositol, it is at the same time almost completely devoid of sodium chloride. More recently, it has been shown by Seamark, Tate \& Smeaton (1968) that inositol is accompanied by another closely related polyol, namely, scillitol (scylloinositol), and that the ratio of inositol to scyllitol in the boar vesicular secretion is approximately $94: 6$. The same investigators have also discovered that in addition to glycerylphosphorylcholine, which is a characteristic secretory product of the boar epididymis (Dawson, Mann \& White, 1957), there is also present in boar seminal plasma some glycerylphosphorylinositol.

It is interesting to note in passing that many of the substances secreted in the male accessory organs of mammals have been originally associated by biochemists with plants rather than animals. This applies to both fructose and citric acid, as well as to glycerylphosphorylcholine which abounds in potatoes, and to ergothioneine whose name recalls its original source, namely, the ergot. Apart from ergothioneine (the betaine of thiolhistidine), which occurs in particularly large amounts in the boar vesicular secretion (Leone \& Mann, 1951; Mann \& Leone, 1953 ), the male accessory fluids of the boar and other mammals contain a great many other nitrogenous bases, the most recent addition to the list of these compounds being hypotaurine (van der Horst \& Grooten, 1966; Grooten, 1967).

For a detailed list of the different chemical substances that have been identified in the secretory fluids produced by the prostate, seminal vesicles, and other male accessory organs, see Mann (1964); Mann \& Lutwak-Mann (1951); Price \& Williams-Ashman (1961); Mann (1973). Here, I would like to describe a few advances mostly relating to species variations and to enzymatic mechanisms responsible for the elaboration of some of the secretory products.

\section{SPECIES VARIATIONS}

In man, a striking feature of the prostatic secretion is the extraordinarily high concentration of citric acid and acid phosphatase. Equally characteristic is the high concentration of fructose in the seminal vesicle secretion. This has been utilized for diagnostic purposes in certain pathological conditions of the human reproductive tract. For instance, conditions such as bilateral occlusion, or 
absence of ejaculatory ducts, which may be encountered in congenital aplasia or in cystic fibrosis, will lead to fructose-free ejaculates of semen. But such ejaculates, although azoospermic and fructose-free, quite often show an abnormally high acid phosphatase activity and citric acid concentration, indicating an excessive proportion of prostatic secretion.

Another peculiarity of the human ejaculate is the exceptionally high concentration of prostaglandins, a group of unsaturated $\mathrm{C}_{20}$-fatty acids characterized by the presence of the cyclopentanone ring. Broadly speaking, the prostaglandins are derived from four rings, designated as E, F, A and B. Thirteen prostaglandins have been detected in human seminal plasma; of these the two most prominent ones are prostaglandin $\mathbf{E}_{1}(11 \alpha, 15$-dihydroxy-9-keto-prosta-13-enoic acid) and prostaglandin $\mathbf{E}_{2}$ (11 $\alpha, 15$-dihydroxy-9-keto-prosta-5,13-dienoic acid) (Bygdeman \& Samuelsson, 1964). So far as is known, the seminal prostaglandins account for most of the smooth-muscle-stimulating activity of human seminal plasma. Their main source is probably the seminal vesicle secretion.

Together, the vesicular and prostatic secretions contribute also the bulk of human seminal plasma proteins. Some of these proteins, however, break down rapidly after ejaculation under the influence of proteolytic enzymes to peptides and amino acids. This is the reason for the rapid decline in protein concentration which occurs in human semen during storage, even at an incubation temperature as low as $+5^{\circ} \mathrm{C}$ (Quinlivan, 1972).

An interesting feature of the secretory activity of male accessory organs in mammals is that, in many instances, organs, which at one time were believed to be 'homologous' by anatomists, differ strikingly in the chemical nature of their secretory products. This is especially true of the secretions of the prostate and seminal vesicle. For example, whereas in man citric acid issecreted by the prostate, and fructose by the seminal vesicles, in other mammalian species the situation is entirely different.

In the boar, for instance, fructose and citric acid are secreted together in the same accessory organ, namely, the seminal vesicle. The chemical composition of the boar accessory secretions differs also in other ways from those of man and other animals. Of special interest in this respect are (i) the high content of glycerylphosphorylcholine and hypotaurine in the epididymis, (ii) the occurrence of ergothioneine, fructose, inositol and citric acid in the seminal vesicles, and (iii) the production of a peculiar sialoprotein containing $26 \cdot 6 \%$ sialic acid by the bulbo-urethral glands (Text-fig. 1). Analyses of these substances in the boar ejaculate provide a very convenient and, at the same time, quantitative indicator of the contribution made by individual accessory organs to the composition of the entire ejaculate. Such analyses have also been useful in determining the sequence in which the various accessory secretions are voided during ejaculation, and in the study of effects of pharmacological agents, particularly atropine, on the secretory functions of the boar reproductive tract (Glover \& Mann, 1954; Dziuk \& Mann, 1963).

Another example of useful application of chemical analyses of accessory secretions to problems in animal reproduction, comes from a recent study of the fate of boar semen after intra-uterine insemination. Using the chemical determinations of citric acid, ergothioneine and fructose as an indicator test, we found 


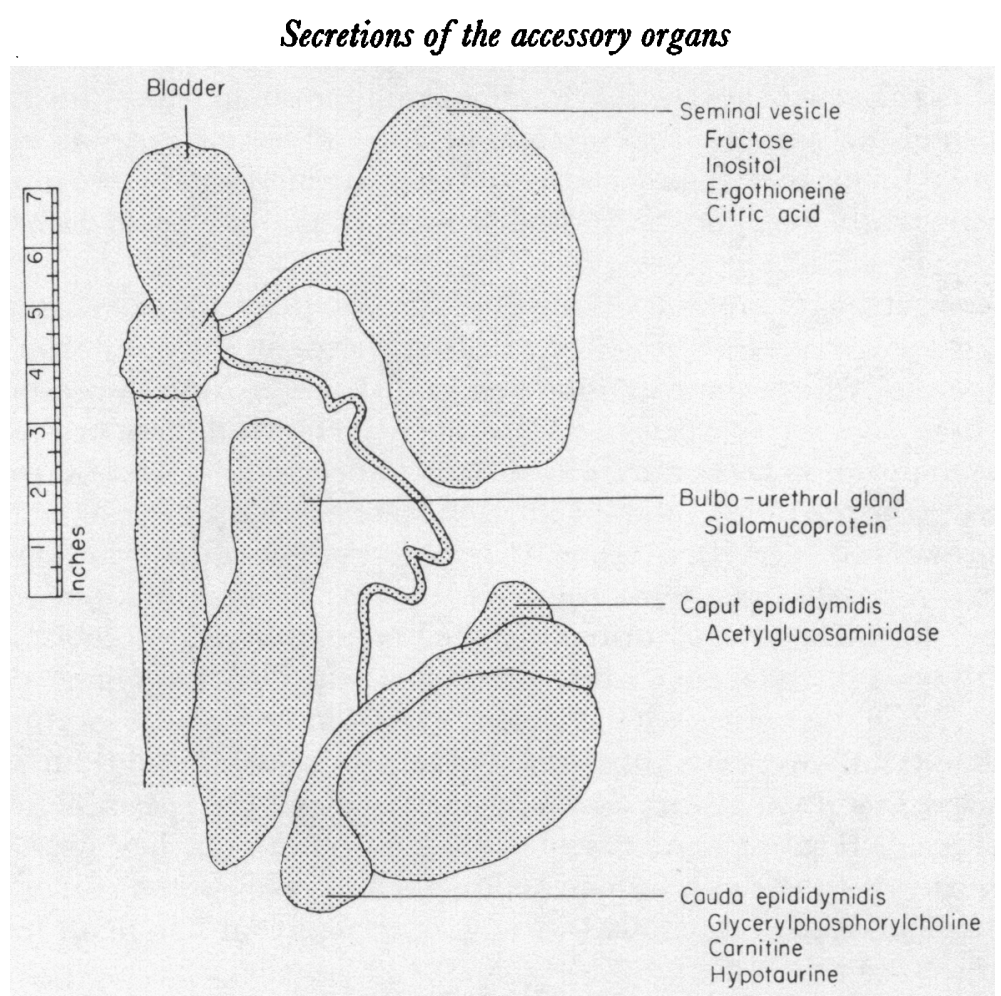

Text-pig. 1. Secretory products of the boar's reproductive tract.

that absorption of seminal plasma constituents from ligated uterine horns, in which boar semen had been deposited artificially, occurs much more slowly than the disappearance of seminal plasma which takes place in the pig after normal mating (Hunter, Karagiannidis \& Mann, 1972). Some other mechanism, possibly the backflow of semen through the cervix, appears to contribute to the rapid post-copulatory disappearance of seminal plasma from the uterus of a naturally mated oestrous pig.

To an even higher degree than in either man or boar, the chemical specificity of the male accessory secretions is apparent in small laboratory animals such as rabbit, mouse or rat. In the rat, the organ specificity, as regards fructose and citric acid formation, is so characteristic that it can be reproduced in transplanted tissues (Lutwak-Mann, Mann \& Price, 1949). If a minute fragment of coagulating gland tissue is transplanted subcutaneously into a female rat, it will grow in response to injected testosterone and will, in due course, produce fructose in a concentration as high as, or higher than, the intact gland. A transplant of the seminal vesicle tissue under similar conditions will produce citric acid, but not fructose.

\section{SECRETORY MEGHANISMS}

Much progress has been made during the last two decades in studies concerned with biosynthetic processes in male accessory organs and with mechanisms by means of which the secretory products pass from these organs into the secretory fluids. 
Concerning the mechanism of fructose formation, so far as is known, mainly from studies of the ram and bull seminal vesicle and the rat coagulating gland, there are two major metabolic pathways, both starting from blood glucose, one proceeding via glucose phosphate and fructose phosphate, and the other via sorbitol.

In the conversion of glucose via sorbitol, the two principal enzymes involved are aldohexose reductase (which reduces glucose to sorbitol) and sorbitol dehydrogenase (which oxidizes sorbitol to fructose); both have been well characterized. In several species, semen has been shown to contain in addition to large amounts of fructose (in human semen about $0.2 \%$ ) small quantities of both glucose (the precursor) and sorbitol (the intermediary).

The biosynthesis of certain other secretory products of the male accessory organs have also received attention in recent years. This applies, among others, to the oldest known organic constituent of the human prostatic secretion, namely, spermine. This is the substance which, in human semen that has been allowed to stand for a while, combines with phosphate to form crystalline spermine phosphate. The crystals were first observed by Leeuwenhoek more than 300 years ago, and were described by him as 'three-sided bodies'. The spermine phosphate crystals are so typical of human semen that, at one time, they were used for forensic purposes to identify human seminal stains. Nowadays it is possible to appreciate their beauty more fully by using sophisticated instruments such as the scanning electron microscope.

Chemically, spermine belongs to the large group of polyamines. As shown by synthesis nearly 50 years ago (Dudley, Rosenheim \& Starling, 1927), it is a base, corresponding in structure to $\alpha, \delta$-bis $\left[\gamma^{\prime}\right.$-aminopropylamino]-butane. Human seminal plasma may contain as much as $0.35 \mathrm{~g}$ spermine $/ 100 \mathrm{ml}$, which is more than any other body fluid or tissue in either man or animals. But some microorganisms and plants are also conspicuously rich in spermine; it is by no means uncommon to encounter spermine in leaves, roots and fruit; and the embryos of cereal grains are known to be capable of accumulating particularly large amounts of spermine (Smith, 1971).

Using the ventral lobes of the rat prostate as their main experimental material, Pegg, Lockwood \& Williams-Ashman (1970) have succeeded recently in elucidating the ways in which the prostate gland manufactures spermine. The key substance in the biosynthesis of spermine is putrescine, a polyamine commonly encountered in the prostate gland, and a well-known metabolite of arginine. There are two ways in which arginine can be converted enzymatically in cells to putrescine, namely, via ornithine or via agmatine, depending on the availability of ornithine decarboxylase or arginine decarboxylase. But so far as the prostate is concerned, putrescine is formed mainly by decarboxylation of ornithine; arginine decarboxylase appears to be absent. Formed in this manner, putrescine interacts with the propylamine residue of decarboxylated S-adenosylmethionine; the latter is formed from S-adenosylmethionine (a product of ATP interaction with methionine) under the influence of a specific enzyme, the Sadenosyl-L-methionine decarboxylase which, as Pegg et al. (1970) have shown, has two unusual properties: it is a putrescine-dependent enzyme, that is, it requires the presence of putrescine for its activity, and it is androgen-dependent, 
that is, its activity decreases rapidly after castration, but is restored by testosterone administration.

The immediate result of the interaction between putrescine and the propylamine residue of decarboxylated adenosylmethionine is spermidine, yet another polyamine commonly found in the prostate and prostatic secretion; spermidine is the immediate precursor of spermine. No separation could so far be effected between the enzyme-forming spermidine from putrescine and the enzymeforming spermine from spermidine.

As regards the physiological rôle of spermine, its direct involvement in the motility or fertilizing function of spermatozoa is unlikely. Certain investigators have stated that the prostatic secretion as a whole has some 'activating' effect on sperm motility, but it is conceivable that this was due to dilution, $\mathrm{pH}$, buffering capacity and similar causes, rather than to the presence of any specific organic substance secreted by the prostate, such as spermine. On the whole, the effects on motility, observed after addition of spermine to sperm suspensions in vitro, have been small (White, 1954; Tabor \& Rosenthal, 1956). As regards the reported positive correlation between spermine concentration and the degree of motility in human ejaculates, one wonders if, in these cases, spermine was not merely an indicator of the contribution made by the prostatic secretion as a whole, i.e. that it was the prostatic secretion in its entirety, rather than spermine alone, that was responsible for the effect on sperm motility (Fair, Clark \& Wehner, 1972).

It has also been suggested that, as spermine is endowed with bacteriostatic properties against certain micro-organisms such as Staphylococcus aureus, it may be responsible for the inhibitory effect which human seminal plasma is known to exert on microbial growth (Gurevitch, Rozansky, Weber, Brzezinsky \& Eckerling, 1951; Grossowicz, Razin \& Rozansky, 1955). But one must bear in mind that human seminal plasma abounds in a variety of proteolytic, nucleolytic and other hydrolytic enzymes some of which may also be capable of interfering with bacterial growth.

One could envisage the possibility that spermine, spermidine, putrescine, ornithine and similar polyamines may exert their physiological effects on the prostate itself, as regards both glandular growth and secretory ability. Polyamines are well known promoters of cellular growth in general. The most recent example that could be cited in that connection (Pohjanpelto \& Raina, 1972) is the identification of putrescine as the factor produced by human fibroblasts in culture, which stimulates the growth and division of these cells. These growth-promoting properties of polyamines are most probably related to their direct involvement in the nucleic acid and protein biosynthesis. Both spermine and spermidine, by virtue of their basic nature, are known to interact readily with acid phosphate groups of deoxyribonucleic acid, and it is conceivable that the 'stabilizing effect', which polyamincs exert on DNA, arises from that type of interaction. Polyamines are also known to be capable of stimulating the activity of the DNA-primed RNA polymerase.

The secretory products of male accessory glands which we have discussed so far are secreted in a soluble form. In addition, male accessory fluids contain certain amounts of so-called particulate matter, which is formed in the course of the secretory processes in the male accessory organs. The two mechanisms in- 
volved are those of apocrine and merocrine activity. By definition, the apocrine mechanism is one in which a portion of the cytoplasm is discharged by the secretory cells together with their secretory products. The merocrine type of secretion, on the other hand, is one in which the glandular cells remain intact during the formation and discharge of their secretory product. Of these two mechanisms, the apocrine is the one largely responsible for the formation of the prostatic and vesicular secretion. This explains why the prostatic and vesicular secretions are rich in enzymes which, in general, one associates with the interior rather than the exterior milieu of cells. That is also why the seminal plasma differs from other body fluids in that it contains several glycolytic enzymes, a variety of nucleases and nucleotidases, and a wide range of lysosomal enzymes, including proteinases, phosphatases, glycosidases and mucolytic enzymes such as, for example, hyaluronidase.

This means that a great many of the macromolecular substances, including enzymes, which abound in prostatic and vesicular secretion, simply represent the contents of secretory cells which have undergone desquamation or disruption during their normal secretory activity. Many of the microscopically visible 'particles' that one normally encounters in ejaculated semen owe their origin to similar causes. In that category are the various so-called 'lipid bodies', 'colostrum corpuscles', 'prostatic calculi' and 'lipid granules', described by various authors in the prostatic secretion and semen of man and animals. The fact that they also occur in ejaculates of vasectomized individuals makes it evident that they are not associated in any way with spermatozoa, but originate in the accessory secretions as such; some of the seminal particles in vasectomized rabbits have been shown to carry antigens closely similar to those that, in the past, have been ascribed to both spermatozoa and seminal plasma (Metz, Hinsch \& Anika, 1968).

\section{PRODUCTS RESULTING FROM INTERACTIONS BETWEEN AN ACCESSORY SECRETION AND SPERMATOZOA, OR BETWEEN TWO ACCESSORY SECRETIONS}

One must always bear in mind the possibility that some of the substances that one normally finds in ejaculated seminal plasma may not have originated in the secretions of the male accessory organs as such, but may have leaked out into the seminal plasma from the spermatozoa. This applies particularly to certain enzymes (Mann, 1964): for example, it was shown recently by Harrison \& White (1972) that spermatozoa or cytoplasmic droplets can both release certain glycolytic enzymes, when semen is rapidly cooled from $30^{\circ} \mathrm{C}$ to $0^{\circ} \mathrm{C}$. It is conceivable that even in fresh seminal plasma some of the glycolytic activity is derived from disintegrating spermatozoa or cytoplasmic droplets.

As knowledge of the biochemistry of male accessory secretions accumulates, new advances are also made concerning the various ways in which the male accessory secretions interact either with each other, or with spermatozoa. A well known type of interaction, in which accessory secretions act upon each other, is the phenomenon of coagulation or gelation of semen. In the rat, coagulation is due to the action of 'vesiculase', an enzyme secreted by the coagulating gland, 
on a coagulable protein secreted by the seminal vesicle; this process results in the formation of the vaginal plug. Quite recently, a similar reaction was described between a coagulinogen secreted by the coagulating gland of the male rat and a coagulating factor present in the uterine fluid of the female rat (Joshi, Yaron \& Lindner, 1972). In the boar, where the seminal gel usually represents a substantial part of the voluminous ejaculate, the formation of the elastic gel is the outcome of an interaction between the sialomucoprotein secreted by the bulbo-urethral glands and at least two specific proteins present in the vesicular secretion (Boursnell, Hartree \& Briggs, 1970).

Among important reactions which involve male accessory secretions and the spermatozoa, one that has been particularly well studied is the action of sperm glycolytic enzymes on the fructose of the seminal plasma, resulting in the formation of lactic acid, and providing the metabolic energy needed for anaerobic survival of spermatozoa. Two other reactions, more recently discovered, concern the transformation of carnitine into acetylcarnitine (Marquis \& Fritz, 1965), and the mechanism which regulates the ratio between the oxidized and reduced form of the nicotinamide adenine dinucleotide coenzyme (NAD) in spermatozoa (Brooks \& Mann, 1971). Carnitine ( $\beta$-oxo- $\alpha$-butyrobetaine) is a nitrogenous base secreted in the rat by the epididymis. Epididymal spermatozoa, owing to the presence of the enzyme carnitine acetyltransferase, act upon carnitine and acetylate it to acetylcarnitine. NAD occurs in fresh spermatozoa in two forms, as $\mathrm{NAD}^{+}$and $\mathrm{NADH}$; the ratio $\mathrm{NAD}^{+} / \mathrm{NADH}$ is controlled by various exogenous substances present in the seminal plasma. Thus, for example, lactic acid produces a rapid conversion of $\mathrm{NAD}^{+}$to $\mathrm{NADH}$, while pyruvic acid produces the opposite effect.

\section{REFERENCES}

Anderson, K. M. \& Liao, S. (1968) Selective retention of dihydrotestosterone by prostatic nuclei. Nature, Lond. 219, 277.

Boursnell, J. C., Hartree, E. F. \& Briggs, P. A. (1970) Studies on the bulbo-urethral (Cowper's) gland mucin and seminal gel of the boar. Biochem. $7.117,981$.

BRooks, D. E. \& MANN, T. (1971) NAD in the metabolism of motile spermatozoa. Nature, Lond. 234, 301.

Bruchovsky, N. \& WiLson, J. D. (1968) The conversion of testosterone to $5 \alpha$-androstan-17 $\beta$-ol-3-one by rat prostate in vivo and in vitro. F. biol. Chem. 243, 2012.

Bygdeman, M. \& SAmuelsson, B. (1964) Quantitative determination of prostaglandin in human semen. Clinica chim. Acta, 10, 566.

Dawson, R. M. C., Mann, T. \& Whrte, I. G. (1957) Glycerylphosphorylcholine and phosphorylcholine in semen, and their relation to choline. Biochem. F. 65, 627.

Dudiey, H. W., Rosenheim, O. \& Starling, W. W. (1927) The constitution and synthesis of spermidine, a newly discovered base isolated from animal tissues. Biochem. 7. 21, 97.

Dziuk, P.J. \& MANN, T. (1963) Effect of atropine on the composition of semen and secretory function of male accessory organs of the boar. F. Reprod. Fert. 5, 101 .

Eliasson, R., Molin, L. \& Rajka, G. (1970) Involvement of the prostate and seminal vesicle in urethritis with special reference to semen analysis. Andrologie, 2, 179.

Fair, W. R., Clark, R. B. \& WeHner, N. (1972) A correlation of seminal polyamine levels and semen analysis in the human. Fert. Steril. 23, 38.

Glover, T. \& ManN, T. (1954) On the composition of boar semen. 7. agric. Sci., Camb. 44, 355.

Grooten, H. J. G. (1967) An investigation into the occurrence of amino acids, chiefly of hypotaurine, and of keto acids in semen and reproductive tract of the boar. Doctoral dissertation, University of Utrecht.

Grossowicz, N., Razin, S. \& Rozanskx, R. (1955) Factors influencing the antibacterial action of spermine and spermidine on Staphylococcus aureus. F. gen. Microbiol. 13, 436.

Gurevitch, J., Rozansky, R., Weber, D., Brzezinsky, A. \& Eckerling, B. (1951) The role of spermine in the inhibition of Staphylococcus aureus by human semen. J. clin. Path. 4, 360. 
Harrison, R. A. P. \& White, I. G. (1972) Glycolytic enzymes in spermatozoa and cytoplasmic droplets of bull, boar and ram, and their leakage after shock. F. Reprod. Fert. 30, 105.

Huggins, C. (1945) The physiology of the prostate gland. Physiol. Rev. 25, 281.

Huggins, C. (1947) The prostatic secretion. Harvey Lect. 42, 148.

Hunter, R. H. F., Karagiannidis, A. \& Mann, T. (1972) Fate of seminal plasma after intrauterine insemination in the pig. 7. Reprod. Fert. 29, 303.

Joshi, M. S., YARon, A. \& LindNeR, H. R. (1972) Intrauterine gelation of seminal plasma components in the rat after coitus. 7 . Reprod. Fert. $30,27$.

Leone, E. \& MANN, T. (1951) Ergothioneine in the seminal vesicle secretion. Nature, Lond. $168,205$.

Lutwax-Mann, C., Mann, T. \& Price, D. (1949) Metabolic activity in tissue transplants. Hormone induced formation of fructose and citric acid in transplants from accessory glands of reproduction. Proc. R. Soc. B, 136, 461.

ManN, T. (1954) On the presence and role of inositol and certain other substances in the seminal vesicle secretion of the boar. Proc. R. Soc. Lond. B, 142, 21.

MANN, T. (1964) The biochemistry of semen and of the male reproductive tract. Methuen, London.

Mann, T. (1973) Biochemistry of semen. In: Handbook of Physiology. Am. Physiol. Soc. (in press).

ManN, T. \& Leone, E. (1953) Studies on the metabolism of semen. 8. Ergothioneine as a normal constituent of boar seminal plasma. Purification and crystallization. Site of formation and function. Biochem. 7. 53, 140.

MANN, T. \& LuTwAK-MANN, C. (1951) Secretory function of the male accessory organs of reproduction in mammals. Physiol. Rev. 31, 25.

Mann, T., Rowson, L. E. A., Baronos, S. \& Karagiannidis, A. (1971) The androgenic potency of dihydrotestosterone and testosterone in the bull, and the effect of growth hormone on the bovine seminal vesicle. 7. Endocr. 51, 707.

Mann, T., Rowson, L. E. A., Short, R. V. \& Skinner, J. E. (1967) The relationship between nutrition and androgenic activity in pubescent twin calves, and the effect of orchitis. 7. Endocr. $38,455$.

Marberger, H., Marberger, E., ManN, T. \& Lutwak-ManN, C. (1962) Citric acid in human prostatic secretion and metastasizing cancer of prostate gland. Br. med. $\mathcal{J}$. i, 835 .

Marquis, N. R. \& Fritz, I. B. (1965) Effects of testosterone on the distribution of carnitine and carnitine acetyltransferase in tissues of the reproductive system of the male rat. 7. biol. Chem. 240, 2197.

Metz, C. B., Hinsch, G. W. \& ANIKA, J. L. (1968) Ultrastructure and antigens of particles from rabbit semen. 7. Reprod. Fert. 17, 195.

Pegg, A. E., Lockwood, D. H. \& Williams-Ashman, H. G. (1970) Concentrations of putrescine and polyamines and their enzymic synthesis during androgen-induced prostatic growth. Biochem. $\mathcal{J}$. $117,17$.

Pohjanpelto, P. \& Raina, A. (1972) Identification of a growth factor produced by human fibroblasts in vitro as putrescine. Nature, New Biol. 235, 247.

Price, D. \& Williams-Ashman, H. G. (1961) The accessory reproductive glands of mammals. In: Sex and Internal Secretions, 3rd edn, vol. 1, p. 366. Ed. W. C. Young. Williams \& Wilkins, Baltimore.

Quinlivan, W. L. G. (1972) The effect of temperature on the proteins of human seminal plasma during storage. Fert. Steril. 23, 163.

Seamark, R. F., TAte, M. E. \& Smeaton, T. C. (1968) The occurrence of scylloinositol and D-glycerol1-(L-myoinositol-1-hydrogen phosphate) in the male reproductive tract. 7. biol. Chem. 243, 2424.

Sirteri, P. K. \& Wilson, J. D. (1970) Dihydrotestosterone in prostatic hypertrophy. I. The formation and content of dihydrotestosterone in the hypertrophic prostate of man. 7 . clin. Invest. 49, 1737.

Smrth, T. A. (1971) The occurrence, metabolism and functions of amines in plants. Biol. Rev. 46, 201.

TABor, C. W. \& Rosenthal, S. M. (1956) Pharmacology of spermine and spermidine. Some effects on animals and bacteria. J. Pharmacol. exp. Ther. 116, 139.

VAN DER Horst, C. J. G. \& Grooten, H. J. G. (1966) The occurrence of hypotaurine and other sulfurcontaining amino acids in seminal plasma and spermatozoa of boar, bull and dog. Biochim. biophys. Acta, 117, 495.

White, I. G. (1954) The effect of some seminal constituents and related substances on diluted mammalian spermatozoa. Aust. 7. biol. Sci. 7, 379.

Williams-Ashman, H. G. \& Reddi, A. H. (1971) Actions of vertebrate sex hormones. A. Rev. Physiol. 33, 31 .

Wilson, J. D. \& Gloyna, R. E. (1970) The intranuclear metabolism of testosterone in the accessory organs of reproduction. Recent Prog. Horm. Res. 26, 309. 